\title{
Adenosine Kinase Expression Modulates Expression of Myelin Proteolipid Protein
}

\author{
Natalie Lai-man Wu and Detlev Boison*
}

\author{
R.S. Dow Neurobiology Laboratories, Legacy Research, 1225 NE 2nd Ave, Portland, OR 97232, USA
}

\begin{abstract}
Adenosine is known to regulate myelination in vitro. Here we tested the hypothesis that adenosine, regulated by adenosine kinase (ADK), might regulate myelin-specific protein expression and myelination in vivo. We demonstrate that transgenic overexpression of ADK, which reduces adenosine in mouse brain, results in increased levels of myelin proteolipid protein.
\end{abstract}

Keywords: Adenosine, transgenic mice, myelination, myelin proteolipid protein.

\section{INTRODUCTION}

Proteolipid protein (PLP), the major protein constituent of CNS myelin has neuroprotective functions and is thought to be responsible for maintaining the integrity of myelinated axons [1, 2]. Thus, mutation [3] or genetic disruption $[4,5]$ of PLP not only affects myelin, but also the integrity and survival of myelinated neurons [2, 6, 7]. Deciphering endogenous mechanisms, which control PLP-expression, is thus of importance for the development of neuroprotective therapies in demyelinating diseases such as multiple sclerosis.

Recent studies suggest that myelination is influenced by neuronal activity. Thus, in an in vitro model of oligodendrocyte maturation and myelination adenosine was identified as a potent neuron-glial transmitter promoting myelination in the CNS in response to action potentials [8]. When added to oligodendrocyte progenitor cell (OPC) cultures, adenosine induced significant morphological changes in OPCs, which subsequently accelerated OPC-axon interactions and myelination at later stages. The diverse array of adenosine receptors, which act through multiple intracellular signaling pathways [9], on OPCs identified in this study [8], opens new areas of research on signals regulating oligodendrocyte development and interactions with neurons.

A recent in vivo study demonstrated that perinatal rodents reared under hypoxia, displayed myelination defects possibly mediated via adenosine $\mathrm{A}_{1}$ receptor activation attributed to an elevation in adenosine levels [10]. This study suggests that adenosine may play different roles in the regulation of myelination of the immature versus the adult brain. Adenosine levels in adult brain are largely regulated by adenosine kinase (ADK), an astrocytic intracellular enzyme that removes adenosine via phosphorylation to AMP and thereby drives the influx of adenosine into the cell $[11,12]$. Due to the existence of a highly active substrate cycle between adenosine and AMP involving ADK and 5'-nucleotidase small changes in ADK activity rapidly translate into major

*Address correspondence to this author at the R.S. Dow Neurobiology Laboratories, Legacy Research, 1225 NE 2nd Ave, Portland, OR 97232, USA; Tel: (503) 413-1754; Fax: (503) 413-5465;

E-mail: dboison@legacyhealthresearch.org changes in ambient adenosine $[13,14]$. Thus transgenic mice with overexpression of brain ADK to $147 \%$ of normal (Adk$\mathrm{tg}$ ) display a reduction of the tone of ambient adenosine in the hippocampal formation [15] resulting in increased susceptibility to seizures [16] and neuronal cell death [17]. In contrast, fb-Adk-def mice with reduced ADK expression in forebrain (62\% of normal) are resistant to acute seizures, acute neuronal injury, and to epileptogenesis due to an increase in the tone of forebrain adenosine [16].

To determine how changes in the adenosine system influence myelination in vivo, we studied myelination and the expression of PLP in Adk-tg and fb-Adk-def mice. Our data show that an increase in ADK expression and therefore reduced adenosine levels results in increased myelin and in increased levels of PLP in adult animals, suggesting that ADK is involved in modulating PLP expression and myelination.

\section{MATERIALS AND METHODS}

\section{Animals}

All animal procedures were conducted in a facility accredited by the Association for Assessment and Accreditation of Laboratory Animal Care in accordance with protocols approved by the Institutional Animal Care and Use Committee and the principles outlined in the NIH Guide for the Care and Use of Laboratory Animals.

\section{Adk Transgenic Mice}

As a tool to study the influence of the adenosine system on myelination in vivo, two lines of Adk mutant mice were generated in our laboratory. Based on an ADK knockout background (i.e. lack of the endogenous astrocytic ADK) [18] a loxP-flanked ADK transgene was introduced with a brain-wide overexpression and a novel neuronal expression of ADK (Adk-tg mice). These mice are characterized by increased neuronal activity and susceptibility to seizures [15] and cell death [17]. The second line of Adk mutant mice was created by forebrain-selective reduction of ADK using transgenic mice expressing Cre recombinase under the control of an Emx 1 promotor [19], which is predominantly expressed in neurons and astrocytes of cortex and hippocampus ( $\mathrm{Fb}$ Adk-def mice) [16]. 


\section{FluoroMyelin ${ }^{\mathrm{TM}}$ Green Fluorescent Stain}

$12 \mu \mathrm{m}$ coronal or sagittal frozen mouse brain cryosections from 21 (P21) or 70 (P70) day old Adk-tg, fb-Adk-def, or wild-type mice were air-dried and fixed in formalin. The slides were washed 3 times in PBT (PBS $+0.2 \%$ Triton X100). Next the sections were stained with FluoroMyelin ${ }^{\mathrm{TM}}$ (Molecular Probes, Eugene, OR) for 20 minutes at room temperature (RT). After that, the slides were rinsed in PBS, and washed 3 times each with PBS. The sections were then mounted with an aqueous antifade-mounting medium for fluorescence microscopy. Images were acquired on a Leica DM LB microscope with the assistance of BIOQUANT NOVA 5.5 image analysis software.

\section{Immunohistochemistry}

For the immunohistochemical detection of myelin proteins, $12 \mu \mathrm{m}$ coronal or sagittal mouse brain cryosections from 21 (P21) or 70 (P70) day old Adk-tg, fb-Adk-def, or wild-type mice were fixed in formalin followed by 3 washes with Tris-buffered saline (TBS). The sections were immersed in an ethanol: acetic acid (95:5) mix for $15 \mathrm{~min}$ at $4^{\circ} \mathrm{C}$, then in $90 \%$ ethanol for $5 \mathrm{~min}$ at $4^{\circ} \mathrm{C}$ and $70 \%$ ethanol for $5 \mathrm{~min}$ at $4^{\circ} \mathrm{C}$. The sections were blocked for $30 \mathrm{~min}$ in TBS, pH 7.4 containing $2 \%$ normal goat serum and $0.2 \%$ Triton X-100 and 1\% bovine serum albumin, and incubated overnight at $4{ }^{\circ} \mathrm{C}$ with primary rabbit anti- PLP or anti-MBP, both diluted 1:100 (Abcam). On the next day, the slides were washed 3 times in TBS; followed by incubation with biotinylated goat anti-mouse/rabbit antibody diluted 1:200 for $2 \mathrm{~h}$ in TBS, pH 7.4, containing 2\% normal goat serum at RT. After washing three times in TBS, the slides were incubated with avidin-biotin enzyme complex (Vectastain Elite Kit; Vector Laboratories, Burlingame, CA). Following 3 washes with TBS, the tissue antigen was localized by incubation with hydrogen peroxide and diaminobenzidine hydrochloride (Sigma, St. Louis, MO), which acts as a chromogen. The sections were washed extensively, air-dried, dehydrated, and coverslipped. The immunoperoxidase-stained sections were analyzed with a Zeiss microscope. The intensity of immunostaining was semi-quantified with a gel-scanning integrated optical density software (Bioquant, Nashville, TN). Regions of the corpus callosum and the overlying cerebral cortex were selected for intensity quantification.

\section{Western Blot Analysis}

Mouse brains ( $n=3$ per genotype and age) from postnatal day 21 and adults (P70) were harvested and cut longitudinally to separate the hemispheres. One of the hemispheres was further sliced sagittally for histology, and the other was used for Western blot analysis. To separate forebrain from hindbrain the hemispheres were cut diagonally from the separation between cortex and cerebellum all the way to the olfactory bulb, thus separating cortex and hippocampus (= "forebrain") from the rest of the hemisphere, which in the following is referred to as "hindbrain". Protein extracts from brain homogenates were prepared, pooled from $n=3$ samples of equal age and genotype and the concentrations were determined by Bradford assay. Protein samples of $2 \mu \mathrm{g} / \mu \mathrm{l}$ were diluted in an equal volume of $2 \times$ sample buffer (125mM Tris/HCl pH 6.8, 20\% glycerol, 0.002\% bromphenol blue, $10 \% \beta$-mercaptoethanol, $4 \%$ SDS) and boiled at $95^{\circ} \mathrm{C}$ for $5 \mathrm{~min} .30 \mu \mathrm{g}$ of protein samples per lane was sub- jected to sodium dodecyl sulfate-polyacrylamide gel electrophoresis (SDS-PAGE). Proteins were transferred onto nitrocellulose membranes using a Trans Blot Mini Cell (Bio Rad). For immunodetection, the membranes were blocked for $1-2 \mathrm{~h}$ in TBST (10mM Tris/HCl $\mathrm{pH} 8,0.15 \mathrm{M} \mathrm{NaCl}, 0.1 \%$ Tween 20) containing $2 \%$ BSA at RT, followed by incubation with primary antibodies (rabbit anti-PLP 1:1000, Abcam, polyclonal rabbit antiserum against $\mathrm{ADK} 1: 4000$, rabbit anti- $\beta$-actin 1:1000, Abcam) overnight at $4{ }^{\circ} \mathrm{C}$ in blocking solution. The blots were washed 3 times with TBST. Incubation with the appropriate secondary antibodies (goat-antirabbit, HRP-conjugated 1:6000 and goat-anti-mouse, HRPconjugated 1:6000) was performed for $1 \mathrm{~h}$ at RT. Following extensive washing immunoreactivity was detected by a commercial chemoluminescence detection kit and exposure of a Kodak X-OMAT film. Images were captured using a Dage 72 camera and immunoreactive bands were quantified with a gel-scanning integrated optical density software (Bioquant, Nashville, TN). Actin immunoreactivity was used to monitor equal sample loading.

\section{RESULTS AND DISCUSSION}

To study whether the adenosine system plays a role in the regulation of myelination during development, we examined the spatio-temporal pattern of myelination in $A d k$ mutant mice. Mutant mice and wild-type control mice were sacrificed at either 21 or 70 days after birth and their brains processed for immunohistochemistry and immunoblotting $(n=3$, each). Each brain was split longitudinally into two hemispheres. One hemisphere was processed for sagittal brain sections, the other for Western Blot analysis.

\section{PLP Expression is Maintained in the Hindbrain of P21 Mutants}

Myelination, including the expression of the two major myelin proteins PLP and myelin basic protein (MBP) [20], of the rodent CNS peaks around postnatal day 21 [21]. We analyzed PLP expression levels by immunoblotting at this time point and compared myelin expression with ADK expression levels in the mutant animals. Adk-tg and fb-Adkdef mice differ exclusively in forebrain ADK expression but, compared to wild type, have comparably elevated levels of ADK in their mid- and hindbrains (Fig. 1a). In line with elevated hindbrain ADK our Western Blot analysis showed a 36 $\%$ increase in PLP expression in the hindbrain of both Adktg and fb-Adk-def mice compared to wild-type controls $(n=$ 3 each). Interestingly, overexpression of PLP was accompanied by increased expression of DM20 an alternative splice product of PLP, which normally is expressed at higher levels during earlier developmental timepoints [22]. At this developmental time point myelination in hindbrain is more advanced than in forebrain, since myelination proceeds in a caudo-rostral direction. Remarkably, we did not find differences in PLP expression in the forebrain of the mutants despite their differences in ADK expression (Fig. 1a), indicating that during ongoing myelination PLP expression is independent of the adenosine/ADK system. Thus, in hindbrain, where myelination is largely completed at P21 [21], PLP levels were increased and DM20 expression was maintained under conditions of increased ADK, suggesting that adenosine may play a role in turning PLP/DM20 expression off during development. 
a

$\underline{\text { Forebrain }} \underline{\text { Hindbrain }}$

$\mathrm{b}$
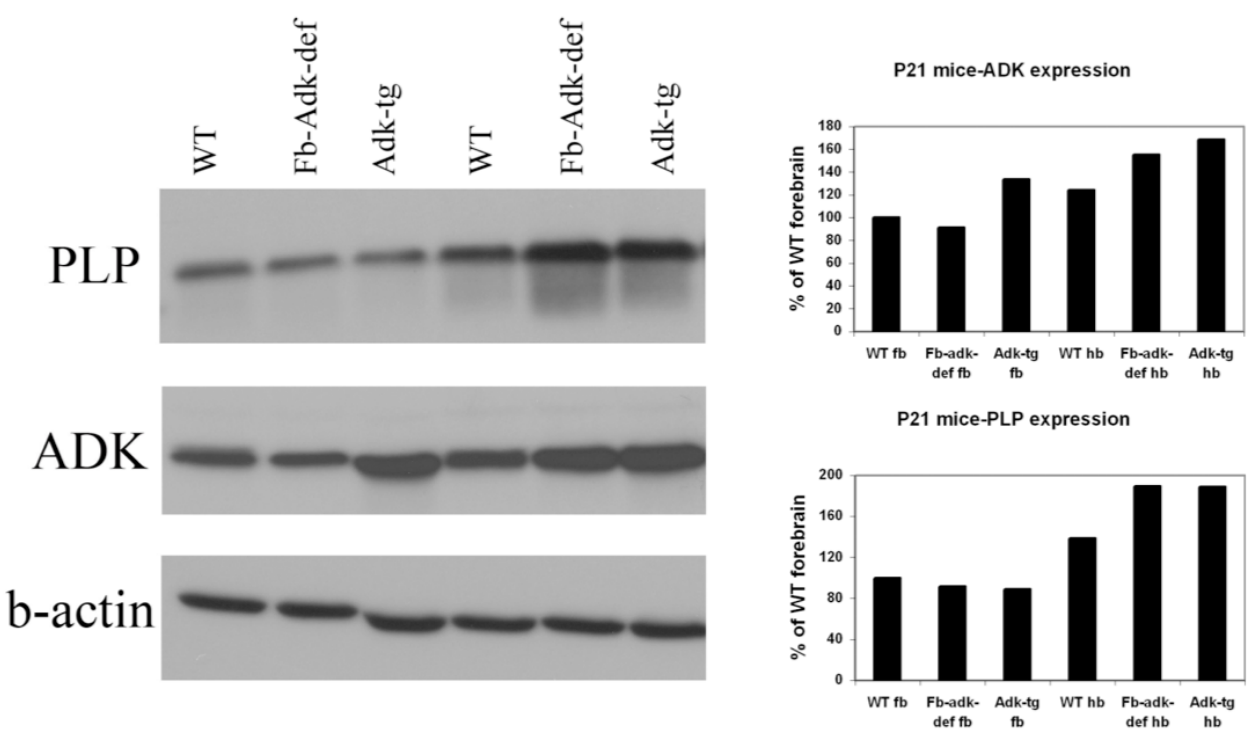

Fig. (1). (a) Western blot analysis of pooled (from $n=3$ samples, each) brain extracts from either forebrain (fb) or hindbrain (hb) of Adk-tg and fb-Adk-def mice at postnatal day 21 using an antibody against the myelin specific protein PLP. Note the presence of the alternative lower molecular splice product DM20 as "smear" beneath the PLP bands in hindbrain. (b) Quantification of protein expression levels. The quantification was based on 3 brains of the same genotype. Pooled samples were analyzed on one Western Blot, normalized first to beta-actin levels and then to WT forebrain. These Western blot data demonstrate that at P21, under conditions of increased ADK in hindbrain of both Adk mutant mice, PLP expression is increased by $36 \%$. However, no difference is detected between the forebrain of the mutants.

\section{PLP Expression is Reduced in Adult Mutant Brains with Reduced Forebrain ADK}

The study of P21 brains indicated that PLP expression and myelination during early postnatal brain development are not regulated by adenosine/ADK; however, the P21 data from hindbrain suggested that PLP expression and myelination are likely modulated by adenosine/ADK in the mature brain. We therefore studied the myelination pattern in adult mutant brains in more detail. In adult brain our Western Blot data revealed increased levels of PLP and DM20 under con-

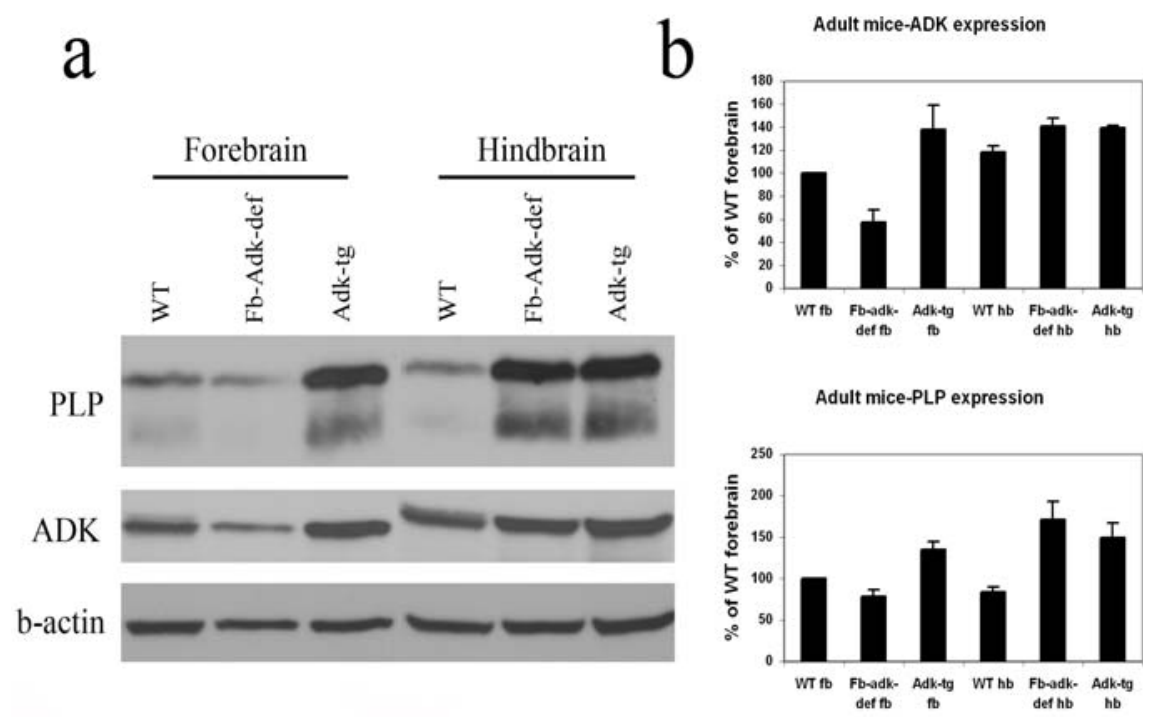

Fig. (2). (a) Western Blot analysis of pooled (from $n=3$ samples, each) brain extracts from either forebrain (fb) or hindbrain (hb) of adult WT, Adk-tg and fb-Adk-def mice. Note high expression levels of the smaller molecular weight isoform DM20 under conditions of high ADK expression. (b) Quantification of protein expression levels as described in Fig. 1, however the experiment was done in duplicate (error bars $=$ SEMs). By immunoblotting, we show a marked increase in PLP expression under conditions of increased ADK expression in mature rodent brains. In forebrain of fb-Adk-def animals PLP reduction reflects ADK reduction. In hindbrain of both mutants, ADK overexpression correlates with increased PLP compared to wild-type controls. 
ditions of transgenic overexpression of ADK (i.e. in forebrain of Adk-tg mice and in hindbrain of Adk-tg and fb-Adkdef mice). In forebrain, ADK expression in fb-Adk-def animals was reduced to $62 \%$ of control, whereas that in Adk-tg mice was $147 \%$ of control (Figs. 2a and 2b). This is consistent with our previous data [17]. Interestingly the expression levels of both PLP and DM20 roughly paralleled those of ADK in adult forebrain. Thus, we found a reduction of PLP expression (78\% of control) in the forebrain of $\mathrm{fb}$-Adk-def animals, while forebrain PLP expression in Adk-tg animals was elevated by $35 \%$ compared to wild-type controls. Consistent with the overexpression of ADK in hindbrain, both mutants had a nearly 2-fold increase in hindbrain PLP-levels.

In addition to the Western Blot analysis described above, we examined the adult mutant brains by histological analy-
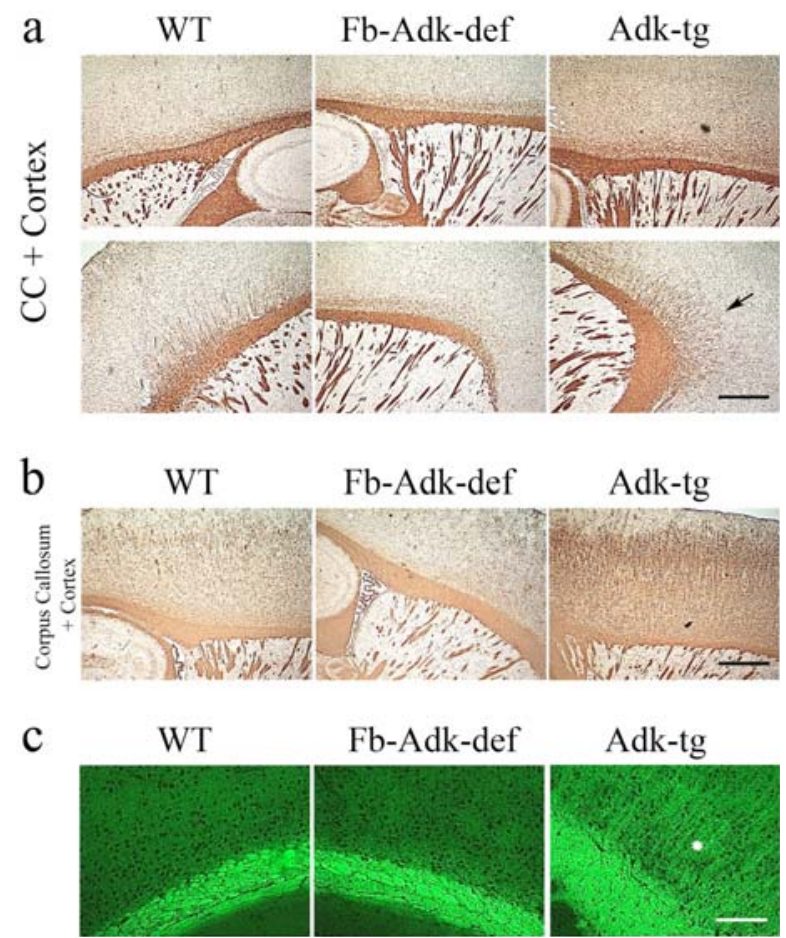

Fig. (3). Histological analysis of myelination in adult wild-type and $A d k$ mutant brains. Photomicrographs illustrate the corpus callosum (CC) and cortical region of the mutant animals. (a) Using an antibody against PLP, we demonstrate an increase in PLP immunoreactivity in the cortical region of Adk-tg mice (intensity of PLP staining: $101.3 \pm 5.1$ RDUs, SEM) compared to wild-type control ( $84.4 \pm 1.3$ RDUs). Note that myelinated fibers in the cortex of Adk-tg mice are longer and denser than wild-type controls (arrow). Fibers in fb-Adk-def brain, however, are barely myelinated $(66.4 \pm 6.2$ RDUs). Scale bar $=300 \mu \mathrm{m}$. (b) Using an antibody against MBP, we demonstrate an increase in MBP immunoreactivity (mean intensity of MBP staining over corpus callosum and the overlying cerebral cortex-WT, 58.9 \pm 5.1 RDUs; FbAdk-tg, 56.9 \pm 2.3 RDUs; Adk-tg, 78.1 \pm 7.8 RDUs), which corresponds to the increase in PLP immunoreactivity shown in (a). Scale bar $=300 \mu \mathrm{m}$. (c) Using a myelin specific stain, FluoroMyelin ${ }^{\mathrm{TM}}$, we demonstrate a remarkable increase in myelin staining in the overlying cerebral cortex of the corpus callosum in Adk-tg brain where ADK levels are elevated. Long myelinated fibers are in high density in the cortex (asterisk). In contrast, the cortex of fb-Adkdef mice is only slightly myelinated. Scale bar $=75 \mu \mathrm{m}$. sis. First, we analyzed brain sections with the anti-PLP antibody using immunohistochemistry. Our data demonstrated an increase in PLP immunoreactivity in normally less myelinated regions of the cortex of Adk-tg mice (i.e. in a region of ADK overexpression) compared to fb-Adk-def mice (with reduced forebrain $\mathrm{ADK}$ ) and wild-type controls. Long myelinated fibers were more abundant and in higher density in the cerebral cortex of Adk-tg mice [mean intensity of PLP staining over corpus callosum and the overlying cerebral cortex, $n=3: 101.3 \pm 5.1$ relative density units (RDU), SEM] compared to wild-type brains ( $84.4 \pm 1.3$ RDUs). In contrast, cortical fibers were scarcely myelinated in the cortex of fb-Adk-def mice (66.4 \pm 6.2 RDUs) (Fig. 3a). Interestingly, changes in PLP expression in dependence of ADK expression, were paralleled by increased expression of myelin basic protein (MBP) suggesting that ADK influences the expression of at least two major myelin constituents.

Next we used a green fluorescent myelin stain to selectively label myelin in brain cryosections derived from adult animals ( $n=3$ per genotype). We found a marked increase of myelin in the forebrain of Adk-tg mice compared to that of fb-Adk-def and wild-type mice (Fig. 3c). The corpus callosum of Adk-tg mouse brain displayed an intense myelin staining. Tangentially oriented cortical fibers of the overlying cerebral cortex, which normally has only moderate amounts of myelin, were highly myelinated in Adk-tg mice, whereas little myelination of these cortical fibers was observed in the $\mathrm{fb}$-Adk-def brains.

\section{CONCLUSION}

Our studies in Adk mutant mice suggest that adenosine/ADK does not influence the expression of PLP during the phase of active de novo myelination. However, when myelination is developmentally completed (in hindbrain around $\mathrm{P} 21$, in forebrain in adults), myelination, and in particular the expression of PLP - and most remarkably, DM20 - is maintained, when ADK levels are high and adenosine levels low. This conclusion is based on three experimental findings reported in this study: (i) PLP/DM20 expression levels correlate with ADK expression levels in Western Blots (reduced PLP in forebrain of adult fb-Adk-def mice; increased PLP in forebrain of adult Adk-tg mice and hindbrain of both mutants); (ii) Histologically, PLP immunoreactivity is decreased in areas of decreased ADK (forebrain of fb-Adk-def mice) and increased in areas of increased ADK (forebrain of Adk-tg mice, hindbrain of both mutants); (iii) The abundance and density of myelinated fibers as seen in the fluoromyelin stain corresponds to expression levels of ADK. These data suggest that adenosine exerts an important influence terminating - rather than initiating - myelination, since reduced adenosine under conditions of high ADK allows additional myelination to occur. Mechanistically, this could be due either to a failure in shutting off the synthesis of PLP or a failure to shut off oligodendrocyte precursor cell activation. Most remarkably, under conditions of high ADK, the expression of the alternatively spliced PLP-isoform DM20 - a marker of immature myelin [22] - is not turned off. Irrespective of the underlying mechanism, our findings suggest that in adult brain, adenosine can modulate the expression of an important myelin protein with putative neuroprotective functions. Thus, therapeutic modulation of adenosine might be a novel strategy to influence PLP-dependent 
neuroprotection. This might be of clinical importance for conditions ranging from multiple sclerosis to stroke. Further investigation is necessary to elucidate the mechanisms of the adenosine / PLP interaction, e.g. in PLP knockout mice. It remains to be demonstrated, e.g. by electron microscopy, whether increases in PLP alter the ultrastructure of myelin, such as the addition of extra-lamellae to myelinated axons or the myelination of un- or hypo-myelinated axons.

\section{ACKNOWLEDGEMENTS}

This work was supported by a grant from the Legacy Research Advisory Committee (RAC). We thank Andrew Wilz for genotyping and maintenance of the mouse colony.

\section{REFERENCES}

[1] Garbern JY. Pelizaeus-Merzbacher disease: Genetic and cellular pathogenesis. Cell Mol Life Sci 2007; 64: 50-65.

[2] Skoff RP, Bessert DA, Cerghet M, et al. The myelin proteolipid protein gene modulates apoptosis in neural and non-neural tissues. Cell Death Differ 2004; 11: 1247-57.

[3] Boison D, Stoffel W. Myelin-deficient rat: a point mutation in exon III (A->C; Thr75 -> Pro) of the myelin proteollipid protein causes dysmyelination and oligodendrocyte death. EMBO J 1989; 8: 3295-302.

[4] Boison D, Büssow H, D'Urso D, Müller H-W, Stoffel W. Adhesive properties of proteolipid protein are responsible for the compaction of CNS myelin sheaths. J Neurosci 1995; 15: 5502-13.

[5] Boison D, Stoffel W. Disruption of the compacted myelin sheath of axons of the central nervous system in proteolipid protein-deficient mice. Proc Natl Acad Sci USA 1994; 91: 11709-13.

[6] Rosenbluth J, Nave KA, Mierzwa A, Schiff R. Subtle myelin defects in PLP-null mice. Glia 2006; 54: 172-82.

[7] Inoue K. PLP1-related inherited dysmyelinating disorders: Pelizaeus-Merzbacher disease and spastic paraplegia type 2. Neurogen 2005; 6: 1-16.

[8] Stevens B, Porta S, Haak LL, Gallo V, Fields RD. Adenosine: a neuron-glial transmitter promoting myelination in the CNS in response to action potentials. Neuron 2002; 36: 855-68.
[9] Fredholm BB, Ijzerman AP, Jacobson KA, Klotz KN, Linden J. International Union of Pharmacology. XXV. Nomenclature and classification of adenosine receptors. Pharmacol Rev 2001; 53: 527-52.

[10] Back SA, Craig A, Luo NL, et al. Protective effects of caffeine on chronic hypoxia-induced perinatal white matter injury. Ann Neurol 2006; 60: 696-705.

[11] Boison D. Adenosine kinase, epilepsy and stroke: mechanisms and therapies. Trends Pharmacol Sci 2006; 27: 652-8.

[12] Boison D. Adenosine as a neuromodulator in neurological diseases. Curr Opin Pharmacol 2007; in press.

[13] Arch JR, Newsholme EA. Activities and some properties of 5'nucleotidase, adenosine kinase and adenosine deaminase in tissues from vertebrates and invertebrates in relation to the control of the concentration and the physiological role of adenosine. Biochem $\mathrm{J}$ 1978; 174: 965-77.

[14] Bontemps F, Van den Berghe G, Hers HG. Evidence for a substrate cycle between AMP and adenosine in isolated hepatocytes. Proc Natl Acad Sci USA 1983; 80: 2829-33.

[15] Fedele DE, Gouder N, Güttinger M, et al. Astrogliosis in epilepsy leads to overexpression of adenosine kinase resulting in seizure aggravation. Brain 2005; 128: 2383-95.

[16] Li T, Ren G, Lusardi T, et al. Adenosine kinase, a target for the prediction and prevention of epileptogenesis. J Clin Inv 2007; in press.

[17] Pignataro G, Simon RP, Boison D. Transgenic overexpression of adenosine kinase aggravates cell death in ischemia. J Cereb Blood Flow Metab 2007; 27: 1-5

[18] Boison D, Scheurer L, Zumsteg V, et al. Neonatal hepatic steatosis by disruption of the adenosine kinase gene. Proc Natl Acad Sci USA 2002; 99: 6985-90.

[19] Iwasato T, Nomura R, Ando R, Ikeda T, Tanaka M, Itohara S. Dorsal telencephalon-specific expression of Cre recombinase in PAC transgenic mice. Genesis 2004; 38: 130-8.

[20] Boggs JM. Myelin basic protein: a multifunctional protein. Cell Mol Life Sci 2006; 63: 1945-61.

[21] Joosten EA, Gribnau AA, Gorgels TG. Immunoelectron microscopic localization of cell adhesion molecule L1 in developing rat pyramidal tract. Neuroscience 1990; 38: 675-86.

[22] Thomson CE, Vouyiouklis DA, Barrie JA, Wease KN, Montague P. Plp gene regulation in the developing murine optic nerve: correlation with oligodendroglial process alignment along the axons. Dev Neurosci 2005; 27: 27-36. 